\title{
Demographic Characteristics of Vitiligo Patients in Beni-Suef University Hospital
}

\author{
Ahmed Ibrahim Mohamed Nagaty ${ }^{a}$, Abd-ElAziz El-Rifaie ${ }^{b}$, Yasser Mostafa Gohary ${ }^{b}$, Laila Ahmed \\ Rashed ${ }^{c}$, Ebtehal Gamal Abdelhady Ghetah ${ }^{d}$ \\ ${ }^{\text {a }}$ Samalot Specialized Centres, El Minia, Egypt \\ ${ }^{\mathrm{b}}$ Dermatology department, Faculty of Medicine, Beni-Suef University, Egypt \\ ${ }^{\mathrm{c}}$ Medical Biochemistry \& Molecular Biology Department, Faculty of Medicine, Cairo University, \\ Egypt \\ ${ }^{\mathrm{d}}$ Medical Biochemistry and Molecular Biology Department, Faculty of Medicine, Beni-Suef \\ University, Egypt
}

\begin{abstract}
Background: Vitiligo is an acquired depigmentary skin disease that manifests as circumscribed depigmented patches or macules on the skin. It may have devastating psychological and social consequences Vitiligo is a common depigmenting disorder with profound psychosocial impacts. Although the etiology of vitiligo remains obscure, but recent experimental studies infer the presence of interactions between melanocytes and other typical skin cells, particularly keratinocytes. Patients and methods: This study investigated the demographic data in 30 vitiligo patients at dermatology department in Beni-Suef University Hospital. Results: The most significant findings in the clinical data were as follow: the female number doubled that of males, $46.7 \%$ of patients were housewives, negative family history of vitiligo was in $85.7 \%$ of patients, the commonest type of vitiligo was the vulgaris $(50.0 \%), 70 \%$ of patients had skin type VI, $80 \%$ of patients had generalized form of disease, $96.7 \%$ had bilateral disease, and $50 \%$ of the biopsies were taken from the back. Conclusion: This was an epidemiologic study of patients with psoriasis in Egypt and provides an overview of the epidemiologic characteristics and clinical profiles of this patient population.
\end{abstract}

Keywords: Vitiligo, Egypt, skin type, VIDA.

\section{Introduction}

Vitiligo is an acquired depigmentary skin disease that manifests as circumscribed depigmented patches or macules on the skin [1].
It may have devastating psychological and social consequences Vitiligo is a common depigmenting disorder with profound psychosocial impacts [2]. Although the etiology 
of vitiligo remains obscure, but recent individuals with hepatic or kidney dysfunction experimental studies infer the presence of [4].

interactions between melanocytes and other typical skin cells, particularly keratinocytes [3].

Vitiligo patients have unpredictable prognosis and response to therapy. However many new therapies have been developed nowadays, but without optimal efficacy. New trends are aiming to understand the etiology of vitiligo which in turn promotes development of new effective therapies [1].

Three main hypotheses explain the pathogenesis of vitiligo that is not exclusive of each other: biochemical/cytotoxic, neural and autoimmune. Oxidative stress and the accumulation of free radicals have been supposed as an important biochemical pathogenic mechanism. Better understanding of the pathogenesis theories may guide us to develop more effective therapy for this common disfiguring disease [4].

Current treatment modalities are directed towards stopping progression of the disease and achieving repigmentation. Therapies include corticosteroids, topical immunomodulators, photo (chemo) therapy, surgery, combination therapies and depigmentation of normally pigmented skin. In photo (chemo) therapy, narrowband ultraviolet-B therapy (NB-UVB) seems to be superior to psoralen ultraviolet-A therapy (PUVA) and broadband UVB. Narrowband UVB (NB-UVB) is a more recent form of phototherapy that uses wavelengths between 305 and $311 \mathrm{~nm}$. NB-UVB can be used in children, pregnant or lactating women and in

\section{Patients and Methods}

This is a study of 30 Egyptian patients with vitiligo at dermatology department, Beni-Suef university hospital. The study was conducted from 2010 to 2015. Patients were diagnosed by detailed history, clinical examination and confirmed by skin biopsies. The history of patients included the following points:

\subsection{Medical history:}

1. Age.

2. Gender.

3. Time of the last new lesion appearance.

4. Occupation.

5. Treatment history.

6. Family history.

\subsection{Clinical assessment to determine:}

1. Vitiligo type.

2. Skin type.

3. Extent of vitiligo.

4. Sites of vitiligo.

5. The vitiligo disease activity score (VIDA) was utilized to evaluate the disease activity.

\subsection{Skin biopsies.}

Four mm punch skin biopsies were taken from patients (vitiligo lesion).

\section{Statistical methodology}

Data were coded and entered using the statistical package SPSS (Statistical Package for the Social Sciences) version 24.

Data was summarized using mean, standard deviation, median, minimum and maximum in quantitative data and using frequency (count) and relative frequency (percentage) for 
categorical data. Comparisons between quantitative variables were done using the nonparametric Kruskal-Wallis and Mann-Whitney tests. For comparison of serial measurements within each patient the non-parametric Wilcoxon signed rank test was used [5]. Correlations between quantitative variables were done using Spearman correlation coefficient [6]. P-values less than 0.05 were considered as statistically significant.

\section{Results}

This study was conducted through analysis of clinical data of 30 patients with confirmed

Table (1): Age, time since the last new lesion, and percentage of involved area of skin of vitiligo.

\begin{tabular}{|c|c|c|c|c|c|}
\hline & \multicolumn{5}{|c|}{ Cases } \\
\cline { 2 - 6 } & Mean & SD & Median & Minimum & Maximum \\
\hline Age & 31.00 & 12.86 & 28.50 & 15.00 & 50.00 \\
\hline Last new lesion (months) & 9.00 & 21.59 & 3.50 & 0.02 & 120.00 \\
\hline$\%$ & 23.63 & 23.94 & 20.00 & 2.00 & 95.00 \\
\hline
\end{tabular}

According to their gender, males represent 10 cases $(33.3 \%)$ while females count 20 (66.7\%). Regarding occupation, fourteen (46.7\%) patients were housewives, two (6.7\%) patients were workers, one (3.3\%) patient worked as a tailor, seven $(23.3 \%)$ of them were students, one (3.3\%) was security, three $(10.0 \%)$ were employees, one $(3.3 \%)$ was a cooker and one (3.3\%) was a carpenter. Regarding family history, four cases have diagnosis of vitiligo referred to the dermatology department, Beni-Suef university hospital during the period between 15 June 2017 and 14 January 2018.

The age of the diseased group ranged between $15-50$ years with mean age of $(31.00 \pm 12.86)$ years. The time at which the last new lesion appeared ranged between 0.02-120.0 months with mean period of $(9.0 \pm 21.59)$ months. The percentage of the affected area of vitiligo skin patients ranged between 2.0-95.0\% with mean percentage of $(23.63 \pm 23.94) \%$. positive family history of vitiligo (14.3\%)

while 24 cases $(85.7 \%)$ have no family history. According to the vitiligo type; eight (26.7\%) cases were of mixed type and fifteen $(50.0 \%)$ cases were vitiligo vulgais, two $(6.7 \%)$ patients were vitiligo universalis, two (6.7\%) patients were segmental, two $(6.7 \%)$ were focal and one $(3.3 \%)$ was acral. According to the skin type; eight cases (26.7\%) were classified as skin type 3 , twenty one cases were type $4(70.0 \%)$ and only one case $(3.3 \%)$ with skin type 5 .

Table (2): Clinical data of vitiligo patients.

\begin{tabular}{|c|c|c|c|}
\hline \multicolumn{2}{|c|}{} & Count & $\%$ \\
\hline Sex & Male & 10 & $33.3 \%$ \\
\hline
\end{tabular}




\begin{tabular}{|c|c|c|c|}
\hline & Female & 20 & $66.7 \%$ \\
\hline \multirow{8}{*}{ Occupation } & Worker & 2 & $6.7 \%$ \\
\hline & Tailor & 1 & $3.3 \%$ \\
\hline & Student & 7 & $23.3 \%$ \\
\hline & Security & 1 & $3.3 \%$ \\
\hline & Housewife & 14 & $46.7 \%$ \\
\hline & Employee & 3 & $10.0 \%$ \\
\hline & Cooker & 1 & $3.3 \%$ \\
\hline & Carpenter & 1 & $3.3 \%$ \\
\hline \multirow[t]{2}{*}{ Family history } & Yes & 4 & $14.3 \%$ \\
\hline & No & 24 & $85.7 \%$ \\
\hline \multirow{6}{*}{ Vitiligo type } & Vulgaris $\backslash$ acrofacial (mixed) & 8 & $26.7 \%$ \\
\hline & Vulgaris & 15 & $50.0 \%$ \\
\hline & Universalis & 2 & $6.7 \%$ \\
\hline & Segmental & 2 & $6.7 \%$ \\
\hline & Focal & 2 & $6.7 \%$ \\
\hline & Acral & 1 & $3.3 \%$ \\
\hline \multirow{3}{*}{ Skin type } & 3 & 8 & $26.7 \%$ \\
\hline & 4 & 21 & $70.0 \%$ \\
\hline & 5 & 1 & $3.3 \%$ \\
\hline
\end{tabular}

Sex

Male Female

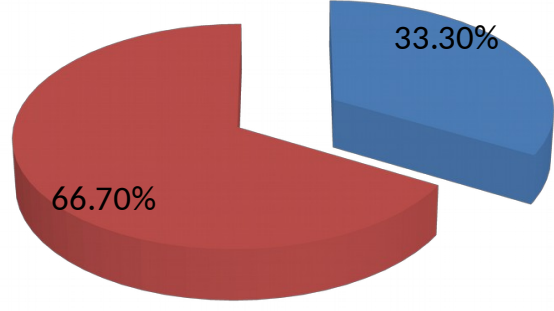

Figure (4): Sex of vitiligo patients.

\section{Family history}

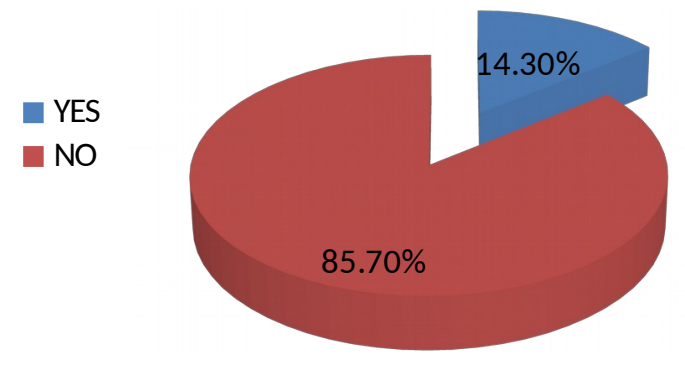

Figure (5): Family history in vitiligo patients.

The distribution of vitiligo was symmetrical in $17(58.6 \%)$ cases and asymmetrical in $12(41.4 \%)$ cases. In

\section{Vitiligo type}
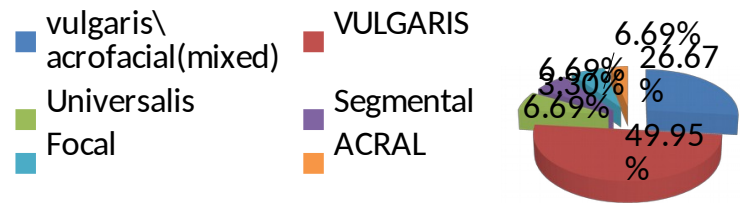

Figure (6): Vitiligo type.

\section{Skin type}

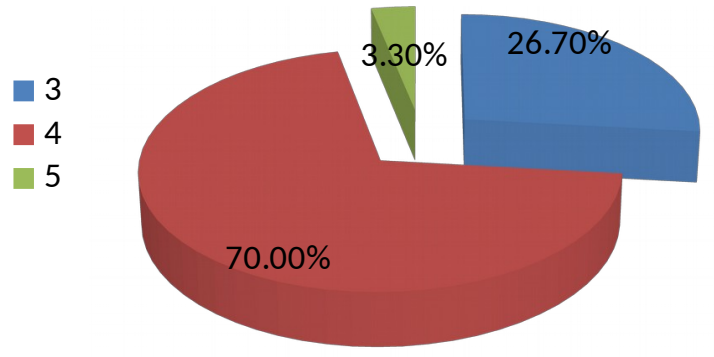

Figure (7): Skin types among vitiligo patients. addition, six $(20.0 \%)$ cases were diagnosed with localized disease while $24(80.0 \%)$ cases 
were diagnosed with generalized form of the disease.

Moreover, twenty nine (96.7\%) cases had bilateral disease and $1(3.3 \%)$ case was unilaterally affected. Sixteen (53.3\%) patients had lesions involving face while the other 14 (46.7\%) patients had lesions that were not involving the face. Hand and feet were affected in $18(60.0 \%)$ patients while the other $12(40.0 \%)$ cases hand and feet were not affected.
Biopsies were harvested from back in 15 $(50.0 \%)$ patients, from leg in $7(23.3 \%)$ patients, from foot in $2(6.7 \%)$ patients and from arm in $3(10.0 \%)$ patients. Other cases were biopsied from thigh in one (3.3\%) case, from shoulder in one $(3.3 \%)$ case, from neck in one $(3.3 \%)$ case.

VIDA was assessed in cases to determine the degree of the disease. Six $(20.0 \%)$ cases were included in stage 0 , two $(6.7 \%)$ cases were in stage $1+$, eight $(26.7 \%)$ cases had stage $2+$, six $(20.0 \%)$ cases were in stage $3+$ and $9(26.7 \%)$ cases were in stage $4+$.

Table (3): Other clinical characteristics of vitiligo patients.

\begin{tabular}{|c|c|c|c|}
\hline & & Count & $\%$ \\
\hline \multirow[t]{2}{*}{ Symmetry } & Symmetrical & 17 & $58.6 \%$ \\
\hline & Asymmetrical & 12 & $41.4 \%$ \\
\hline \multirow[t]{2}{*}{ Local/generalized } & Localized & 6 & $20.0 \%$ \\
\hline & Generalized & 24 & $80.0 \%$ \\
\hline \multirow[t]{2}{*}{ Bilateral/ unilateral } & Unilateral & 1 & $3.3 \%$ \\
\hline & Bilateral & 29 & $96.7 \%$ \\
\hline \multirow[t]{2}{*}{ Face } & Yes & 16 & $53.3 \%$ \\
\hline & No & 14 & $46.7 \%$ \\
\hline \multirow[t]{2}{*}{ Hand and feet } & Yes & 18 & $60.0 \%$ \\
\hline & No & 12 & $40.0 \%$ \\
\hline \multirow{7}{*}{ Biopsy } & Thigh & 1 & $3.3 \%$ \\
\hline & Shoulder & 1 & $3.3 \%$ \\
\hline & Neck & 1 & $3.3 \%$ \\
\hline & Leg & 7 & $23.3 \%$ \\
\hline & Foot & 2 & $6.7 \%$ \\
\hline & Back & 15 & $50.0 \%$ \\
\hline & Arm & 3 & $10.0 \%$ \\
\hline \multirow{5}{*}{ VIDA } & 0 & 6 & $20.0 \%$ \\
\hline & $1+$ & 2 & $6.7 \%$ \\
\hline & $2+$ & 8 & $26.7 \%$ \\
\hline & $3+$ & 6 & $20.0 \%$ \\
\hline & $4+$ & 8 & $26.7 \%$ \\
\hline
\end{tabular}

Figure (8): Symmetry of lesions distribution in vitiligo patients. 


\section{Localized/ generalized}

\section{Localized} genaralized

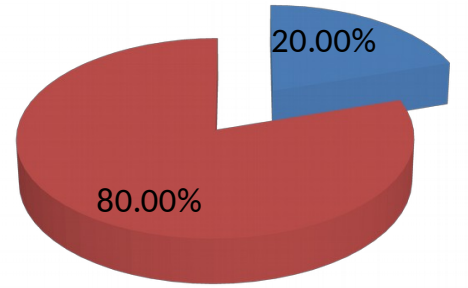

Figure (9): Classification of vitiligo into localized and generalized.

\section{Bilateral/ unilateral}

- Unilateral

- Bilateral

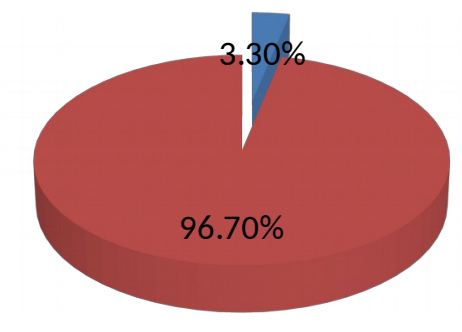

Figure (10): Vitiligo disease; unilateral or bilateral.

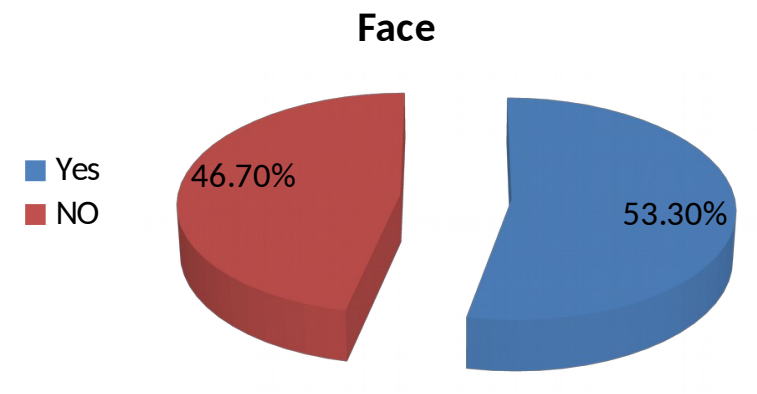

Figure (11): Face involvement in vitiligo patients.
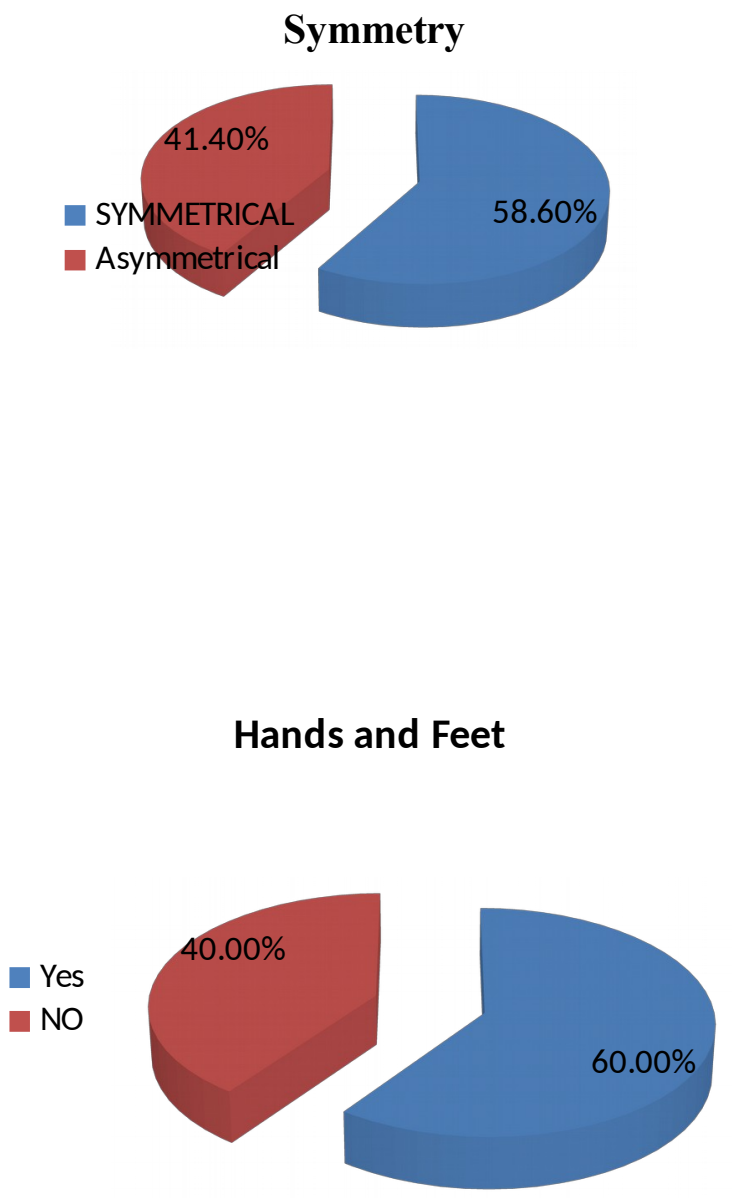

Figure (12): Hands and feet involvement in vitiligo patients.

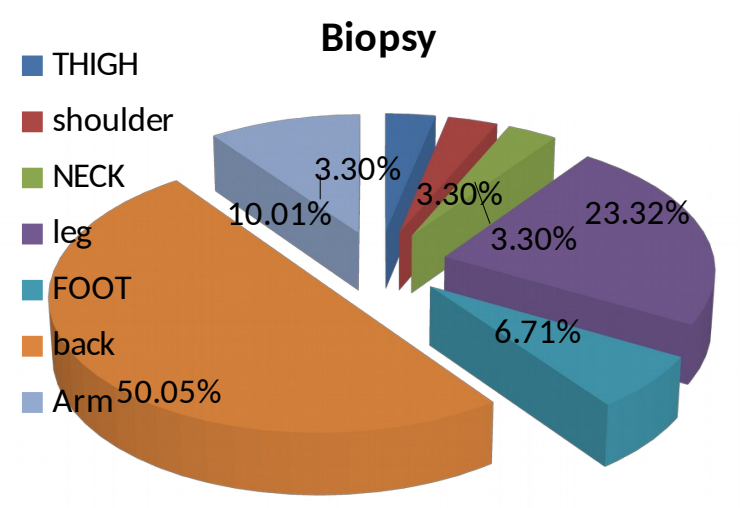

Figure (13): Biopsy sites in vitiligo patients. 


\section{VIDA}

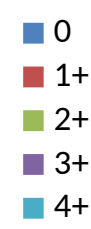

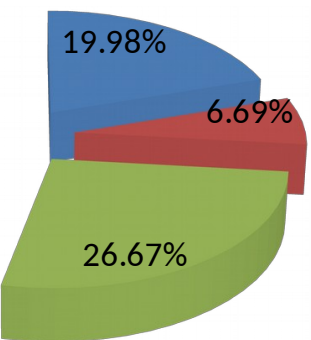

\section{Discussion}

Vitiligo is the most prevalent pigmentary disorder, which has a variety of comorbidities [7]. Vitiligo is a chronic, acquired, common, often heritable, progressive depigmenting disorder, which mainly affects melanocytes from epidermis basal layer [8]. It is characterized by the appearance of wellcircumscribed milky cutaneous macules devoid of identifiable melanocytes on the skin and mucous membranes [9]. Although non-fatal in nature, vitiligo causes severe negative psychosocial impact on the affected individuals, such as social stigmatization and decreased quality of life [10].

Vitiligo is a multifactorial disorder, related to both genetic and non genetic factors. It is generally agreed that there is an absence of functional melanocytes in vitiligo skin due to their destruction. Several hypostheses have been proposed to explain the pathophysiology of melanocytes destruction [11]. However, there is strong evidence that vitiligo is an autoimmune disease [12].

The disfigurement associated with vitiligo could cause serious emotional stress for the

Figure (14): VIDA in vitiligo patients

patient and affect his quality of life. Sun protection of vitiliginous areas with sun blocks is important to prevent sunburn, photodamage and occurrence of Koebner phenomenon [13], [14]. Successful treatment of vitiligo is often difficult, and treatment modalities have traditionally centered on a combination of camouflage products, topical corticosteroids, topical immunomodulators, psoralen plus ultraviolet A light therapy (PUVA) and ultraviolet B (UVB) light therapy. Successful repigmentation occurs only for approximately half of treated patients, leaving many patients without a solution [15].

The aim of this work was to study the various vitiligo types, distribution of disease, family history, and to find out age, sex, occupation of vitiligo patients.

As regards clinical data, the present study results showed that the age of vitiligo patients ranged from 15 to 50 years with average age of 31 years, consistent with the other reports; a study operated on 246 vitiligo cases, the average age was 25.9 years old and the age range 15 to 24 years was most affected $29.3 \%$, and $82.9 \%$ of patients was under 40 years old [16], and also 
with a study reported that the mean age of 34.5 years in 69 vitiligo patients [17]. However, one study included 74 vitiligo patients; their age ranged from 5 to 68 years although their mean age was 31.5 years almost as the present study [18].

In the present study, the time at which the last new lesion appeared ranged between 0.02120.0 months with mean period of $(9.0 \pm 21.59)$ months. In addition, the percentage of the affected area of vitiligo skin patients ranged between 2.0-95.0\% with mean percentage of $(23.63 \pm 23.94) \%$. In another study performed on 613 vitiligo patients had less than $25 \%$ body surface area involved [19]. Moreover, another study found that the mean of the extent of disease (the affected \% of body) was $22 \pm 19.40$ $\%$ in 74 vitiligo patients [18].

The female to male ratio in this study was $2: 1$; males are 10 cases (33.3\%) while females count $20(66.7 \%)$. Most of the other reports shown that males and females were affected with almost in the same percentages, for instance, a previous study showed that among 150 recruited vitiligo patients, $103(68.7 \%)$ were women and $47(31.3 \%)$ were men [20]. This was different from a study that showed, male $131(53.3 \%)$ was more commonly affected than female $115(46.7 \%)$ [16].

The number of female vitiligo patients were found to be higher than male because women notice the change in appearance and approach the doctors sooner than men and of the social stigma in the community, young females tend to report earlier due to matrimonial anxiety [19].

According to the present study, housewife is the most common occupation found among vitiligo patients, followed by students, employees, workers, other jobs as tailor, cooker, carpenter, security were at the bottom. This was similar to a previous study that documented housewife $(33.0 \%)$ as the most frequent occupation, followed by office job (30.4\%), production $(7.4 \%)$, student $(6.8 \%)$, private business $(4.3 \%)$, health care/medical (3.2\%), agriculture $(2.6 \%)$, construction $(2.1 \%)$, and transportation (1.7\%). Beauty shop, fishery, mining/manufacturing, athlete, soldier, and painter were combined into 'others' [23]. Whereas another study found that between 150 vitiligo patients; homemakers (34\%) were the commonest included job followed by student (13.3\%), professional (11.3\%), and sales, manual laborer (41.3\%) [20].

A positive family history of vitiligo was present in $14.3 \%$ of cases in this stidy, while $85.7 \%$ cases have no family history. Similar percentages of positive family history were reported in previous studies [18, 24]. But, another study showed that cases with negative family history of vitiligo were more than positive ones [17].

Vitiligo vulgaris $(50.0 \%)$ was most common type observed in our study followed by mixed, then by universalis, segmental, focal, and finally acral which is similar with some previous studies [16, 19], while different from other studies $[17,21]$. 
According to the present study; the most encountered skin type was type 4 , followed by type 3 , then skin type 5, and this was in consistent with a previous study [20], but in contrast to another study that reported skin type 3 as the most common one among vitiligo patients [22].

In the present study, the distribution of vitiligo was symmetrical in $58.6 \%$ of cases and asymmetrical in $41.4 \%$ of cases, twenty percentages had localized disease while $80.0 \%$ had generalized form of the disease, and $96.7 \%$ showed bilateral disease and $3.3 \%$ was unilaterally affected. This agreed with a previous study that found that majority of patients had bilateral distribution [19].

The results of the current study showed that $53.3 \%$ patients had lesions involving face, and $60.0 \%$ showed hands and feet were affected. Biopsies were harvested mostly from back, followed by leg, and then foot, arm, thigh, shoulder, and neck. This was in accordance to a study that documented $41.1 \%$ of cases with hands or feet involvement, and $55.1 \%$ with facial involvement [23].

In the present study, VIDA was assessed in cases to determine the degree of the disease finding that stage $2+$ and stage $4+$ were the commonest, followed by stage 0 and stage $3+$, and finally stage +1 . In a previous study, it was reported that among 74 vitiligo patients, VIDA score ranged from 0 to 4 , with mean value of $2.40[18]$.
6. Conclusion and Recommendations

Although we attempted to reveal multilateral aspects of the clinical profiles of Egyptian patients with vitiligo, due to the recruitment of adult patients from Beni-Suef university hospitals, the representation of the general vitiligo population in Egypt is limited. Further, because this study was crosssectional, it is necessary to use caution when interpreting results for causal relationships between disease- related factors and outcomes over time. Therefore, additional research in the form of prospective longitudinal cohort studies is needed to further understanding of vitiligo in the Egyptian population.

\section{References}

1- Manga P, Elbuluk N and Orlow SJ. Recent advances in understanding vitiligo. F1000Research. Faculty of 1000 Ltd, 2016; 5: 2234 .

2- Nguyen CM, Beroukhim K, Danesh MJ, et al. The psychosocial impact of acne, vitiligo, and psoriasis: a review. Clin Cosmet Investig Dermatol. Dove Press, 2016; 9: 383-92.

3- Becatti M, Fiorillo C, Barygina V, Cecchi C, et al. SIRT1 regulates MAPK pathways in vitiligo skin: insight into the molecular pathways of cell survival. Journal of cellular and molecular medicine, 2014; 18(3): 514529.

4- Borderé AC, Lambert $\mathrm{J}$ and Van Geel N. (2009): Current and emerging therapy for the management of vitiligo. Clinical, 
cosmetic and investigational dermatology: CCID, 2009; 2: 15.

5- Chan YH. Biostatistics 102: quantitative data-parametric and non-parametric tests. Blood pressure, 2003a; 44(8): 391396.

6- Chan, YH. Biostatistics 104: correlational analysis. Singapore Medicine Journal, 2003b; 44(12): 614-619.

7- Wolff K, Goldsmith L, Katz S, Gilchrest B, et al. Fitzpatrick's dermatology in general medicine, 2008; I: 616-621

8- Ahn JJ, Lee JH, Shin MK and Lee MH. Two Cases of Vitiligo Developed on the Persisting Dermal Melanocytosis: Is There a Difference between Epidermal Melanocytes and Dermal Melanocytes? Annals of dermatology, 2013; 25(2): 226-228.

9- Singh U, Singh S and Pandey SS. Study of Serum Immunoglobulin Levels: Complements $\mathrm{C} 3$ and $\mathrm{C} 4$ in Vitiligo Patients in Northern India. Prospects in Bioscience, 2012; 111-115.

10- Yu R, Huang $\mathrm{Y}$, Zhang $\mathrm{X}$ and Zhou $\mathrm{Y}$. Potential role of neurogenic inflammatory factors in the pathogenesis of vitiligo. Journal of cutaneous medicine and surgery, 2012; 16(4): 230-244.

11-Bilal A and Anwar I. Guidelines for the management of vitiligo. Journal of Pakistan Association of Dermatology, 2016; 24(1): $68-78$

12-Sandoval-Cruz M， García-Carrasco M, Sánchez-Porras R, Mendoza-Pinto $\mathrm{C}$, et al. Immunopathogenesis of vitiligo.
Autoimmunity reviews, 2011; 10(12): 762765.

13-Burns T, Breathnach S, Cox $\mathrm{N}$, et al Textbook of dermatology. Blackwell, 2004; 49: 32-49.

14- Lebwohl MG, Heymann WR, Berth-Jones J, et al. Treatment of Skin Disease: Comprehensive Therapeutic Strategies, $2^{\text {nd }}$ edit Mosby Elsevier, Philadelphia, USA, 2006; 683-687.

15- Patel NS, Paghdal KV and Cohen GF. Advanced treatment modalities for vitiligo. Dermatologic Surgery, 2012; 38(3): 381-391.

16-Dégboé B, Atadokpèdé F, Saka B, Adégbidi $\mathrm{H}$, et al. Vitiligo on black skin: epidemiological and clinical aspects in dermatology, Cotonou (Benin). International journal of dermatology, 2017; 56(1): 92-96.

17-Alenizi DA. Consanguinity pattern and heritability of Vitiligo in Arar, Saudi Arabia. Journal of Family and Community Medicine, 2014; 21(1): 13-16.

18- Rashed L, Hay RA, Mahmoud R, Hasan N, Zahra A, et al. Association of AngiotensinConverting Enzyme (ACE) gene polymorphism with inflammation and cellular cytotoxicity in vitiligo patients. PloS one, 2015; 10(7): e0132915.

19- Vora RV, Patel BB, Chaudhary AH, Mehta, $\mathrm{MJ}$, et al. A clinical study of vitiligo in a rural set up of Gujarat. Indian journal of community medicine: official publication of 
Indian Association of Preventive \& Social Medicine, 2014; 39(3): 143-146.

20-Morales-Sánchez, MA, Vargas-Salinas M, Peralta-Pedrero ML, Olguín-García MG, et al. (2017): Impact of Vitiligo on Quality of Life. Actas dermo-sifiliograficas, 2017; 108(7): 637-642.

21-Bakry OA, Hagag MM, Kandil MA and Shehata, WA. Aquaporin 3 and E-Cadherin Expression in Perilesional Vitiligo Skin. Journal of clinical and diagnostic research: JCDR, 2016; 10(12): WC01WC06.

22-Van Geel N, Lommerts JE, Bekkenk MW, Prinsen CA, et al. Development and validation of a patient-reported outcome measure in vitiligo: The Self-Assessment Vitiligo Extent Score (SA-VES). Journal of the American Academy of Dermatology, 2017; 76(3): 464-471.

23- Jeon IK, Park CJ, Lee, MH, Lee DY, et al. A Multicenter Collaborative Study by the Korean Society of Vitiligo about Patients' Occupations and the Provoking Factors of Vitiligo. Annals of dermatology, 2014; 26(3): 349-356. 